\title{
Nutritious Semisolid Food Gel with Phenethyl Isothiocyanate
}

National Cancer Institute

\section{Source}

National Cancer Institute. Nutritious Semisolid Food Gel with Phenethyl Isothiocyanate.

NCI Thesaurus. Code C148137.

A proprietary, flavored, semisolid food gel-based nutritional supplement containing a variety of vitamins, iron, calcium, protein, fat and carbohydrates, in combination with the phytochemical phenethyl isothiocyanate (PEITC), that can be consumed for nutritional support, and with chemopreventive and anti-cancer activities. Upon oral intake, the nutritious semisolid food gel may aid in the prevention of malnutrition and weight loss. The food gel has a semisolid texture and is specifically formulated for patients who have difficulty chewing and swallowing. PEITC may delay cancer growth, and inhibit cancer cell migration and invasion. 